\title{
Estratégias de aprendizagem e fatores motivacionais relacionados
}

\section{Learning strategies and related motivational factors}

\author{
Liliane Ferreira Neves Inglez de Souza ${ }^{1}$
}

\begin{abstract}
RESUMO
O estudo sobre aprendizagem autorregulada de uma perspectiva sociocognitiva tem integrado fatores cognitivos, metacognitivos, afetivos e motivacionais. Um dos constructos mais investigados nesta área é o de estratégias de aprendizagem, pois seu uso pelos estudantes é considerado um importante indicativo do nível de autorregulação. Nota-se que embora muitos alunos conheçam um repertório de estratégias, nem sempre relatam utilizá-las com frequência. Assume-se, portanto, que esta atividade requer esforço e é marcadamente afetada pela motivação do aluno. Dessa forma, tem-se como finalidade neste artigo, discutir a relação entre estratégias de aprendizagem e algumas variáveis motivacionais que têm sido sistematicamente relacionadas a este constructo: autoeficácia, metas de realização e valor da tarefa.
\end{abstract}

Palavras-chave: estratégias de aprendizagem; motivação para aprendizagem; crenças motivacionais; auto-eficácia; valor da tarefa.

\begin{abstract}
The study about self-regulated learning from a socio-cognitive perspective has integrated cognitive, metacognitive, affective and motivational aspects. Learning strategy has been one of the most investigated constructs in this area, because its use by students is considered a relevant indicative of the self-regulation level. It has been noted that although many students know different strategies, they do not relate to use them frequently. Therefore,
\end{abstract}

1 Doutora em Educação na área de Psicologia, Desenvolvimento Humano e Educação pela Universidade Estadual de Campinas. Professora da Universidade Paulista e da Faculdade de Administração e Artes de Limeira. E-mail: lfneves@terra.com.br. 
it is assumed that this activity requires effort and is markedly affected by students' motivation. For that reason, this article aims to discuss the relation between learning strategies and some motivational variables that have been systematically related to this construct: self-efficacy, achievement goals and task-value.

Keywords: learning strategies; achievement motivation; motivational beliefs; self-efficacy; task-value.

\section{Introdução}

A aprendizagem no contexto escolar é mediada por fatores diversos, não podendo ser atribuído exclusivamente às capacidades cognitivas do aluno, o sucesso ou insucesso neste processo. Nesse sentido, tem se considerado a importância de se investigar tanto a dimensão cognitiva quanto afetivo-motivacional do desempenho escolar. Embora o estudo dos aspectos motivacionais esteve, por um tempo, relativamente negligenciado (WEINER, 1979), particularmente nas duas últimas décadas essa temática vem tomando um lugar de importância nas pesquisas, especialmente no sentido de entender como a motivação afeta a cognição e a aprendizagem (PINTRICH, 2003).

O processo de ensino-aprendizagem é hoje entendido como uma construção que envolve um papel ativo por parte do aluno. Nesta perspectiva, torna-se imprescindível que o aluno desenvolva a capacidade de estabelecer as próprias metas, planejar e monitorar seus esforços na direção de um melhor desempenho acadêmico, direcionando em certa medida, sua aprendizagem no contexto escolar.

Nessa direção, Boekaerts (1996) propôs que o ensino adequado não é mais visto como transferir informação para a memória do estudante e a aprendizagem adequada também não é mais igualada a ter bons resultados nas avaliações. Ao contrário, muitos pesquisadores defendem a visão de que um dos maiores objetivos da educação formal deveria ser equipar os estudantes com capacidades autorregulatórias. Assim são considerados aprendizes autorregulados aqueles que possuem capacidades adaptativas e estratégias para alcançar demandas de mudança (MC CORMICK, 2003, p. 81).

Nos estudos sobre aprendizagem autorregulada convergem fatores afetivomotivacionais, cognitivos e metacognitivos. Porém, nenhum constructo tem sido mais discutido e investigado do que as estratégias de aprendizagem. De modo geral, a literatura sobre o tema tem apontado que o uso de estratégias está condicionado a uma série de fatores, sendo muitos destes de natureza motivacional. 
Assim, este artigo tem o objetivo de discutir alguns aspectos motivacionais dos estudantes e suas relações com o uso das estratégias de aprendizagem.

\section{Estratégias de Aprendizagem}

De modo geral, uma estratégia de aprendizagem envolve diversos recursos utilizados pelos estudantes ao aprender um novo conteúdo, ou desenvolver determinadas habilidades, podendo ser abrangente e generalizável à aprendizagem de várias tarefas e conteúdos ou restrita a uma tarefa específica. De acordo com Lopes da Silva e Sá (1993) as estratégias de aprendizagem podem ser definidas em um nível maior de complexidade como "planos formulados pelos estudantes para atingirem objetivos de aprendizagem e, em um nível mais específico, como qualquer procedimento adotado para a realização de uma determinada tarefa” (p. 19). Reforçando esta afirmação, Paris e Winograd (1990) propuseram que as estratégias podem ser específicas, como usar um algoritmo para checar contas em Matemática ou gerais, como planejar e monitorar uma tarefa.

É possível verificar que as estratégias de aprendizagem abrangem uma gama ampla de ações e, dessa forma, existem muitas maneiras de classificá-las de acordo com diferentes autores. Por exemplo, Danserau (1985) diferenciou entre estratégias primárias que são aquelas ligadas diretamente à execução das tarefas (por exemplo, as estratégias mnemônicas) e as estratégias de apoio que ajudam o aprendiz a estabelecer as condições apropriadas para estudar (por exemplo: planejamento, controle da atenção, monitoramento, dentre outras).

Embora sejam muitas as formas de entender as diferentes estratégias, a classificação mais empregada atualmente é aquela que distingue as estratégias entre cognitivas e metacognitivas. Segundo Dembo (1994), as estratégias cognitivas se referem a comportamentos e pensamentos que propiciem que a informação seja armazenada mais eficientemente (por exemplo: ensaio, elaboração, organização). Já as estratégias metacognitivas constituem procedimentos que o indivíduo usa para planejar, monitorar e regular seu próprio pensamento.

De modo geral, conclui-se que as estratégias cognitivas estão diretamente relacionadas à execução de tarefas, ao passo que as estratégias metacognitivas geralmente são mais amplas e implicam na organização, regulação e mesmo na avaliação do uso das estratégias cognitivas. Ressalta-se ainda que o monitoramento pode informar se uma estratégia em particular está promovendo resultados efetivos e se o estudante está alcançando níveis de desempenho adequados aos seus padrões internos.

Assim, pode-se afirmar que as estratégias permitem planejar e monitorar 
o próprio desempenho. Dessa forma, Lopes da Silva e Sá (1993) apontaram que muitas dificuldades de aprendizagem podem ser explicadas pela ausência ou uso inapropriado de estratégias de estudo e pela inexistência de hábitos favoráveis à aprendizagem.

Essa afirmação é complementada por Derry (1990) ao propor que os estudantes que conhecem e sabem utilizar estratégias são mais bem preparados para lidar com uma variedade de situações de aprendizagem. Nesse sentido, tanto professores como alunos deveriam estar cientes da existência de diferentes estratégias, sua aplicabilidade e relevância na aprendizagem do aluno.

Burkell et al. (1990) acrescentaram que um aluno estratégico, além de possuir uma variedade de estratégias direcionadas a um desafio cognitivo, é capaz de avaliar se estas produzem progresso em direção aos objetivos estabelecidos. Um outro aspecto da relevância de estratégias é seu caráter compensatório. Garner e Alexander (1989) apontam para a existência de um consenso sobre a relevância do conhecimento anterior do aluno em facilitar ou dificultar a aquisição de um novo conteúdo. Contudo, o uso de diferentes estratégias pelo aluno pode ajudar a compensar lacunas em seu conhecimento prévio.

A partir dos pontos levantados anteriormente, é possível listar uma série de benefícios relacionados ao uso de estratégias para o processo de aprendizagem, os quais se refletem até mesmo no desempenho escolar (SCHLIEPER, 2001; COSTA, 2000). No entanto, nem sempre os alunos as conhecem ou utilizam com a finalidade de melhorar a aprendizagem e, por isso, é importante ressaltar que sua utilização está condicionada a uma série de fatores.

Nota-se com frequência que algumas estratégias são usadas espontaneamente pelos alunos sem a necessidade de instrução direta, ao passo que outras, embora ensinadas, raramente são utilizadas. Uma explicação plausível para este fato, de acordo com Garner e Alexander (1989), são as diferenças na motivação dos alunos. Reforçando essa afirmação, a partir de uma revisão na literatura sobre o tema, Costa e Boruchovitch (2000) levantaram alguns pontos que influenciam o uso de estratégias e dentre estes, destacam-se variáveis motivacionais em geral, crenças sobre inteligência, autoeficácia, idade e série escolar dentre outros aspectos.

Eccles e Wigfield (2002) apontaram ainda que teóricos da motivação estão cada vez mais interessados pelas maneiras em que motivação e cognição atuam juntas. Há teóricos, por exemplo, que se dedicam a estudar as conexões entre motivação e o uso de várias estratégias cognitivas.

Como o uso sistemático de estratégias requer esforço, o aprendiz só verá sentido em utilizar diferentes estratégias de aprendizagem quando tiver percepção da sua relevância e quando se julgar capaz de produzir os efeitos desejados. Dessa forma, pode-se inferir que variáveis motivacionais estão diretamente 
relacionadas ao uso de estratégias. Assim, esses aspectos são compreendidos mais como um suporte no uso de estratégias do que como uma influência direta sobre o desempenho acadêmico (MCWHAW; ABRAMI, 2001).

\section{Fatores Motivacionais}

Conforme apontado, uma das questões centrais no estudo das estratégias de aprendizagem é o que motiva os estudantes a utilizá-las. Na tentativa de compreender esta questão, muitos pesquisadores têm procurado descrever as relações entre estratégias e constructos motivacionais, pois embora muitos alunos possuam capacidades autorregulatórias, nem sempre se envolvem em atividades direcionadas a este fim. Ressalta-se que essa atividade requer esforço e, portanto, deve estar relacionada ao interesse ou necessidade do aluno em aprender. Burkel et al. (1990) acrescentam ainda que os estudantes estão mais propensos a usar estratégias se eles têm consciência que procedimentos estratégicos de fato melhoram a performance.

A importância atribuída tanto a fatores cognitivos quanto motivacionais é evidenciada por Zimmermam e Bandura (1994, p. 846) ao afirmarem que “uma coisa é possuir capacidades autorregulatórias e outra coisa é conseguir aplicá-las persistentemente em face de dificuldades, fatores estressantes ou interesses paralelos”.

Muitas crenças pessoais exercem influência na motivação e no desempenho do estudante, sejam estas autorreferenciadas, ou relacionadas ao objeto do conhecimento. Tratando desse aspecto, Pintrich (1999) apontou que embora haja um número grande de variáveis motivacionais, seus estudos juntamente com seus colaboradores haviam se centrado em três tipos de crenças: autoeficácia, valor da tarefa e tipos de metas. Esses conceitos serão discutidos a seguir.

\section{Orientações motivacionais e metas de realização}

De acordo com a teoria de metas de realização (AMES; ARCHER, 1988), há dois grandes grupos de metas estabelecidas pelos alunos: meta aprender, predominantemente relacionada à tarefa, na qual os alunos geralmente são intrinsecamente motivados, tendo como principal objetivo aperfeiçoar os conhecimentos, e meta performance, na qual o aluno busca primordialmente evidenciar capacidades ou esconder lacunas nas mesmas.

As metas afetam as ações, portanto, a cada conjunto de metas se atribuem 
diferentes comportamentos. A meta aprender é em geral relacionada à escolha de tarefas desafiadoras, maior esforço despendido, valorização do próprio progresso, domínio e inovação nas atividades de aprendizagem (BZUNECK, 1999). Ames e Archer (1988) afirmam ainda que com a meta aprender "agrega-se importância ao desenvolvimento de novas habilidades. O processo de aprendizagem em si é valorizado e a obtenção de maestria é vista como sendo dependente do esforço.” (p. 260). Em síntese, o aluno está mais preocupado com suas realizações do que suas notas escolares.

Já na meta performance, busca-se obter notas altas, superando os demais colegas e demonstrando o próprio valor através de comparações sociais. O estudante orientado a esse tipo de meta evita demonstrar que não domina um assunto e, portanto, escolhe tarefas mais fáceis e menos desafiadoras. Complementando, Ames e Archer (1988) apontam que "com uma orientação à meta performance há uma preocupação em ser julgado capaz e mostrar evidência de habilidade tendo sucesso, tendo melhor desempenho que outros, conseguindo sucesso com pouco esforço” (p. 260). É válido ressaltar, conforme Bzuneck (1999), que o termo performance representa o desempenho conforme percebido e avaliado ou julgado externamente.

Quanto à associação entre estratégias e metas, Pintrich (1999) apontou que foram encontradas relações muito consistentes entre esses constructos, sendo que, em geral, a literatura tem mostrado que os alunos mais orientados à meta aprender relatam um uso mais efetivo de estratégias tanto cognitivas quanto autorregulatórias ou metacognitivas (AMES; ARCHER, 1988; ABLARD; LIPSCHULTZ, 1998; WOLTERS; PINTRICH, 1998; MCKHAW; ABRAMI, 2001; CARDOSO; BZUNECK, 2004).

Uma orientação a esta ou àquela meta pode estar vinculada a experiências pessoais na família, nos grupos sociais e também marcadamente na escola. Nesse aspecto, o papel da estrutura escolar foi evidenciado no estudo de Shih e Alexander (2000), que pesquisaram o impacto do tipo de feedback oferecido aos alunos acerca de seu desempenho nas avaliações. Os resultados apontaram que informações socialmente comparadas estavam vinculadas a um maior desenvolvimento da meta performance, ao passo que feedback autorreferenciado vinculava-se a uma orientação maior à meta aprender.

\section{Crenças de autoeficácia}

Dentre as crenças autorreferenciadas, a autoeficácia é um dos constructos mais relacionados ao desempenho escolar e ao uso de estratégias, e pode ser definida como a crença das pessoas acerca de suas capacidades para alcançarem determinadas realizações ou desempenhos (BANDURA, 1986; 1997). Deve-se 
ressaltar que esta não se refere especificamente à capacidade de um indivíduo, mas sim ao que o mesmo acredita ser capaz de realizar, em uma variedade de circunstâncias. Acrescenta-se que a autoeficácia constitui um importante mecanismo de agência, pois muitas ações exercidas no ambiente são reguladas pelas crenças dos indivíduos em suas capacidades para exercê-las (BANDURA, 1992).

A autoeficácia é formada a partir de quatro fontes de informação: experiências de êxito, experiências vicariantes, persuasão social e estados afetivos ou fisiológicos (BANDURA, 1997). Dessa forma, tem se tornado evidente o papel da escola em promover não somente capacidades cognitivas, mas favorecer o desenvolvimento de crenças autorreferenciadas favoráveis para sustentar o uso dessas capacidades.

Como resultado de diversos estudos que têm confirmado as formulações teóricas, é possível afirmar que essas crenças têm uma influência na ação, na motivação e nos processos cognitivos, sendo estes últimos, relacionados à antecipação de consequências e resultados de ações. Aplicando-se o conceito ao contexto escolar, entende-se que a autoeficácia pode afetar a motivação dos alunos para realizar as tarefas ou evitá-las, as reações dos estudantes diante de suas realizações e até mesmo as suas escolhas profissionais. Essa é uma importante justificativa para que diversos estudos tenham sido conduzidos, relacionando este constructo ao desempenho escolar (DOBARRO, 2007; INGLEZ DE SOUZA; BRITO, 2008).

Uma revisão da literatura a respeito da autoeficácia acadêmica, especialmente na década de 1990, apontou que as pesquisas tendem a confirmar a hipótese de que percepções positivas do aluno quanto à própria capacidade afetam a persistência do aluno e o uso de estratégias que tornam a aprendizagem mais eficaz. Estudantes que demonstram maiores crenças de autoeficácia são capazes de persistir mais em face de dificuldades (PAJARES, 1996; PAJARES; MILLER, 1994), aceitam tarefas mais desafiadoras, limitam menos suas escolhas (O'BRIEN; MARTINEZ-PONZ; KOPALA, 1999; BANDURA; BARBARANELLI; CAPRARA; PASTORELLI, 2001) e experimentam níveis mais baixos de ansiedade (BANDALOS; YATES; THORNDIKE-CHRIST, 1995). Além disso, há evidência de que a autoeficácia influencie a autorregulação da aprendizagem (ZIMMERMAN; MARTINEZ-PONS, 1990; WOLTERS; PINTRICH, 1998; BOUFFARD-BOUCHARD; PARENT; LARIVÉE, 1991; INGLEZ DE SOUZA, 2007).

Respectivamente ao uso de estratégias, acredita-se que a importância da autoeficácia resida no fato de que, diante de expectativas favoráveis ao próprio desempenho, estudantes tendem a despender maior esforço e persistir até encontrar as estratégias adequadas para a solução de tarefas, especialmente aquelas mais complexas. 


\section{Valor da tarefa}

Wigfield e Eccles (2000) propuseram que as escolhas, a persistência e o desempenho dos indivíduos podem ser explicados por suas crenças sobre quão bem podem se sair em determinada tarefa, bem como pela quantidade de valor atribuído à atividade em questão.

O valor da tarefa envolve crenças sobre a importância, interesse e peso de determinada tarefa ou disciplina do contexto escolar. Complementando, Wigfield (1983) apontou que o valor da tarefa possui três componentes, a saber: utilidade, importância e interesse. Eccles e Wigfield (2002) afirmaram que o valor de utilidade é determinado pela relação da tarefa com objetivos atuais e futuros, tais como interesses em carreiras profissionais.

Pintrich (1999) apontou ainda que a percepção do indivíduo sobre a utilidade da tarefa pode incluir crenças de que o curso lhe será imediatamente útil de alguma maneira ou em seus futuros estudos e na carreira em geral. Nessa direção, alguns estudos apontaram que as crenças de valor da tarefa estavam positivamente correlacionadas com o uso de estratégias cognitivas como pesquisar, elaborar e organizar. Nos estudos de Wolters e Pintrich (1998) e Pintrich (1999), apontou-se que o valor da tarefa estava vinculado a um maior uso de estratégias e ao desempenho dos alunos, embora esta relação tenha sido mais modesta do que a encontrada nas crenças de autoeficácia.

O valor da tarefa também foi apontado como fonte de motivação, pois "se não se percebe a utilidade do que se deve aprender, o interesse e o esforço tendem a diminuir à medida que o aluno se pergunta para que serve saber o que se pretende que aprenda" (TAPIA; MONTERO, 2004, p. 179). Portanto, perceber a utilidade das disciplinas escolares pode ser decisivo em despertar o interesse do aluno, direcionando esforços para a autorregulação da aprendizagem.

\section{Implicações}

Os resultados das pesquisas revisadas no presente artigo sugerem claramente que algumas crenças motivacionais são mais adaptativas que outras e ajudam a promover e sustentar a aprendizagem autorregulada. Esses dados trazem algumas implicações: por um lado se mostra que alunos mais estratégicos efetivamente atingem um melhor rendimento no processo de aprendizagem. Por outro lado, mostra-se que usar estratégias é uma tarefa que pode exigir esforço e persistência, portanto requer um padrão motivacional adequado. 
Embora para os estudiosos da temática, estejam claros os benefícios do uso de estratégias, em alguns estudos nacionais, apontou-se que existe pouca diversidade nas estratégias mencionadas pelos estudantes; além do que, as estratégias apontadas não são usadas com muita frequência (SCHLIEPER, 2001; INGLEZ DE SOUZA, 2007).

Acredita-se que para promover a aprendizagem autorregulada, é esperado que os alunos conheçam um repertório de estratégias e estejam dispostos a usá-las, além de se sentirem confiantes de que podem ter bons resultados na aprendizagem. Diante desse quadro, conclui-se que ao menos duas ações são necessárias: o ensino de estratégias e a promoção de crenças motivacionais adaptativas.

Infelizmente, no contexto nacional, se tem notícia de poucas iniciativas nesse sentido. Aparentemente, as raras intervenções neste sentido ocorrem em pesquisas de delineamento experimental (por exemplo: JALES, 1997; RIOS, 2005).

Ao se pensar sobre instrução de estratégias, emergem questões sobre quais estratégias devem ser ensinadas, de que maneira e em que contexto se deve fazê-lo, além do papel do professor nesse processo. Valdés (2003) apontou que a discussão sobre como se devem ensinar estratégias de aprendizagem segue duas tendências: a existência de programas paralelos às disciplinas e a instrução dentro de cada uma das disciplinas. De acordo com a autora, o primeiro enfoque tem recebido críticas, e a principal delas é que habilidades desenvolvidas à margem do conteúdo curricular nem sempre são transferidas, pelos alunos, aos objetivos do processo pedagógico. Dessa forma, alguns autores (Burkell et al., 1990; Valdés, 2003) têm defendido a ideia de que a instrução de estratégias seja incorporada ao currículo, não acontecendo exclusivamente de forma paralela ao ensino-aprendizagem das disciplinas.

A partir de uma revisão sobre vários programas de instrução, Paris, S. G. e Paris, A. H. (2001) mencionaram uma série de características de intervenções bem sucedidas que envolveram ações como: fornecer uma rica variedade de estratégias que os estudantes podem usar em tarefas acadêmicas; procurar esclarecer como, quando e porque os alunos devem aplicar estratégias; envolver os colegas na modelação; estimular a transferência das estratégias ensinadas para outros domínios; contextualizar a estratégia em atividades diárias, ajudando os estudantes a perceberem sua utilidade nas atividades curriculares.

Um ponto relevante sobre a instrução de estratégias é que não basta simplesmente ensinar um inventário de estratégias, pois os alunos precisam compreender como e porque usá-las. Um programa de instrução deve envolver uma conscientização acerca da importância das estratégias, salientando os aspectos motivacionais relacionados ao seu uso. Um outro ponto crucial, para 
que um programa de instrução seja bem sucedido, é levar em conta o contexto de sala de aula e, nesse sentido, é necessário que os professores encorajem os alunos a serem estratégicos. Levando em conta esses aspectos, as intervenções podem ser efetivas no sentido de melhorar o uso de estratégias, desenvolvendo a capacidade de autorregulação por parte dos estudantes, no que se refere aos seus processos de aprendizagem.

\section{REFERÊNCIAS}

ABLARD, K. E.; LIPSCHULTZ, R. E. Self-regulated learning in high achieving students: relations to advanced reasoning, achievement goals, and gender. Journal of Educational Psychology, v. 90, p. 94-101, 1998.

AMES, C.; ARCHER, J. Achievement goals in the classroom: students' learning strategies and motivation processes. Journal of Educational Psychology. v. 80, n. 3, p. 260-267, 1988.

BANDALOS, D. L.; YATES, K.; THORNDIKE-CHRIST, T. Effects of mathematics self-concept, perceived self-efficacy, and attributions for failure and success on test anxiety. Journal of Educational Psychology, v. 87, n. 4, p. 611-623, 1995.

BANDURA, A. Social foundations of thought and action: a social cognitive theory. Englewood Cliffs: Prentice-Hall, 1986.

Exercise of personal agency through the self-efficacy mechanism. In: SCHWARZER, R. (Ed.). Self-efficacy: thought control and action. USA: Taylor and Francis, 1992.

. Self-efficacy: the exercise of control. New York: Freeman, 1997.

BANDURA, A.; BARBARANELLI, C.; CAPRARA, G. V.; PASTORELLI, C. Selfefficacy beliefs as shapers of children's aspirations and career trajectories. Child Development, v. 72, n. 1, p. 187-206, 2001.

BOEKAERTS, M. Self-regulated learning at the junction of cognition and motivation. European Psychologist, v. 1, n. 2, p. 100-112, 1996.

BOUFFARD-BOUCHARD, T.; PARENT, S.; LARRIVEE, S. Influence on Selfregulation and performance among junior and senior high-school age students. International Journal of Behavioral Development, v. 14, p. 153-164, 1991. 
BURKELL, J. et al. Cognitive strategy instruction. Cambridge: Brookline Books, 1990.

BZUNECK, J. A. Uma abordagem sócio-cognitivista à motivação do aluno: a teoria de metas de realização. Psico-USF, v. 4, n. 2, p. 51-66, 1999.

CARDOSO, L.; BZUNECK, J. A. Motivação no ensino superior: metas de realização e estratégias de aprendizagem. Psicologia Escolar e Educacional, v. 8, n. 2, p. 145155, 2004.

COSTA, E. R. As estratégias de aprendizagem e a ansiedade de alunos do ensino fundamental: implicações para a prática educacional. Dissertação (Mestrado em Educação) - Faculdade de Educação, Universidade Estadual de Campinas. Campinas, 2000.

COSTA, E. R.; BORUCHOVITCH, E. Fatores que influenciam o uso de Estratégias de Aprendizagem. Psico-USF, v. 5, n. 1, p. 11-24, 2000.

DANSERAU, D. F. Learning strategy research. In: SEGAL, J. W.; CHIPMAN, S. F.; GLASER, R. (Orgs.). Thinking and learning skills. Hillsdale: L. Erlbaum, 1985.

DEMBO, M. H. Applying educational psychology. New York: Longman, 1994.

DERRY, S. J. Learning strategies for acquiring useful knowledge. In: JONES, B. F.; IDOL, L. (Orgs.). Dimensions of thinking and cognitive instruction. Hillsdale: Lawrence Erlbaum Associates, 1990.

DOBARRO, V. R. Solução de problemas e tipos de mente matemática: relações com as atitudes e crenças de auto-eficácia. Tese (Doutorado em Educação) - Faculdade de Educação, Universidade Estadual de Campinas. Campinas, 2007.

ECCLES, J. S.; WIGFIELD, A. Motivational beliefs, values and goals. Annual Review of Psychology, v. 53, p. 109-132, 2002.

GARNER, R.; ALEXANDER, P. A. Metacognition: answered and unanswered questions. Educational Psychologist, v. 24, n. 2, p. 143-158, 1989.

INGLEZ DE SOUZA, L. F. Auto-regulação da aprendizagem e a Matemática escolar. Tese (Doutorado em Educação) - Faculdade de Educação, Universidade Estadual de Campinas. Campinas, 2007.

INGLEZ DE SOUZA, L. F.; BRITO, M. R. F. Crenças de auto-eficácia, autoconceito e desempenho em matemática. Estudos de Psicologia, Campinas, v. 25, n. 2, p. $193-$ 201, 2008.

JALLES, C. M. C. R. O efeito de instruções sobre estratégias metacognitivas de 
crianças pré-escolares em solução de problema geométrico: um estudo exploratório. Dissertação (Mestrado em Educação) - Faculdade de Educação, Universidade Estadual de Campinas. Campinas, 1997.

LOPES DA SILVA, A.; SÁ, I. Saber estudar e estudar para saber. Porto: Porto, 1993.

MCCORMICK, C. B. Metacognition and learning. In: REYNOLDS, W. M.; MILLER, G. E. Handbook of Psychology. 2003. v. 7: Educational Psychology.

MCWHAW, K.; ABRAMI, P. C. Student goal orientation and interest: effects on students' use of self-regulated learning strategies. Contemporary Educational Psychology, v. 26, p. 311-329, 2001.

O'BRIEN, V.; MARTINEZ-PONS, M.; KOPALA, M. Mathematics self-efficacy, ethnic identity, gender, and career interests related to mathematics and science. The Journal of Educational Research, v. 92, n. 4, p. 232-236, 1999.

PAJARES, F. Self-Efficacy beliefs and mathematical problem-solving of gifted students. Contemporary Educational Psychology, v. 21, p. 325-344, 1996.

PAJARES, F.; MILLER, M. D. Role of self-efficacy and self-concept beliefs in mathematical problem solving: a path analysis. Journal of Educational Research, v. 86, n. 2, p. 193-203, 1994.

PARIS, S. G.; WINOGRAD, P. How metacognition can promote academic learning and instruction. In: JONES, B. F.; IDOL, L. Dimensions of thinking and cognitive instruction. Hillsdale: Lawrence Erlbaum Associates, 1990.

PARIS, S. G.; PARIS, A. H. Classroom applications of research on self-regulated learning. Educational Psychologist, v. 36, n. 2, p. 89-101, 2001.

PINTRICH, P. R. The role of motivation in promoting and sustaining self-regulated learning. International Journal of Educational Research, v. 31, p. 459-470, 1999.

. Motivation and classroom learning. In: REYNOLDS, W. M.; MILLER, G. E. Handbook of Psychology. 2003. v. 7: Educational Psychology. p. 103-124.

RIOS, E. R. C. A intervenção por meio da instrução em estratégias de aprendizagem: contribuições para a produção de textos. Tese (Doutorado em Educação) - Faculdade de Educação, Universidade Estadual de Campinas. Campinas, 2005.

SCHILIEPER, M. D. M. J. As estratégias de aprendizagem e as atribuições de causalidade de alunos do ensino fundamental. Dissertação (Mestrado em Educação) - Universidade Estadual de Campinas. Campinas, 2001. 
SHIH, S.; ALEXANDER, J. M. Interacting effects of goal setting and self- or otherreferenced feedback on children's development of self-efficacy and cognitive skill within the Taiwanese classroom. Journal of Educational Psychology, v. 92, p. 536543, 2000.

TAPIA, J. A.; MONTERO, I. Orientação motivacional e estratégias motivadoras na aprendizagem escolar. In: COLL, C. et al. Desenvolvimento psicológico e educação. Porto Alegre: Artmed, 2004.

VALDÉS, M. T. M. Estrategias de aprendizaje: bases para la intervención psicopedagógica. Revista de Psicopedagogia, v. 20, n. 62, p. 136-142, 2003.

WEINER, B. A theory of motivation for some classroom experiences. Journal of Educational Psychology, v. 71, n. 1, p. 3-25, 1979.

WIGFIELD, A.; ECCLES, J. S. Expectancy-Value Theory of Achievement Motivation. Contemporary educational Psychology, v. 25, p. 68-81, 2000.

WOLTERS, C. A.; PINTRICH, P. R. Contextual differences in student motivation and self-regulated learning in mathematics, English, and social studies classrooms. Instructional Science, v. 26. p. 27-47, 1998.

ZIMMERMAN, B. J.; BANDURA, A. Impact of self-regulatory influences on writing course attainment. American Educational Research Journal, v. 31, p. 845-862, 1994.

ZIMMERMAN, B.; MARTINEZ-PONZ, M. Construct validation of student selfregulated learning. Journal of Educational Psychology, v. 80, p. 284-290, 1988.

ZIMMERMAN, B.; MARTINEZ-PONZ, M. Student differences in self-regulated learning: relating grade, sex, and giftedness to self-efficacy and strategy use. Journal of Educational Psychology, v. 82, p. 51-59, 1990.

Texto recebido em 19 de novembro de 2009.

Texto aprovado em 12 de janeiro de 2010. 